\section{La structure des mucines conditionne les propriétés viscoélastiques des gels de mucus}

Bastien Demouveaux ${ }^{1}$, Valérie Gouyer ${ }^{1}$, Mylène Magnien ${ }^{1}$, Ségolène Plet ${ }^{1}$, Frédéric Gottrand ${ }^{1}$, Tetsuharu Narita ${ }^{2,3}$, Jean-Luc Desseyn ${ }^{1}$

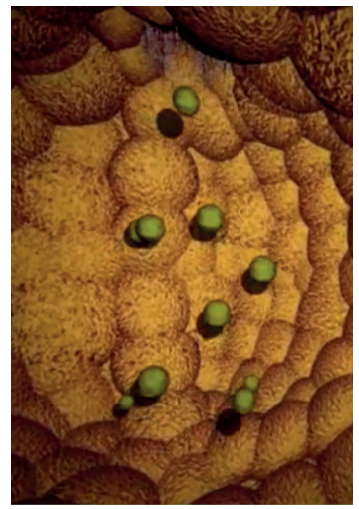

${ }^{1}$ Inserm, Université de Lille, CHU Lille, LIRIC UMR 995, F-59000 Lille, France.

${ }^{2}$ PSL Research University, UPMC Univ. Paris 06, ESPCI Paris, CNRS UMR 7615, Laboratoire Sciences et Ingénierie de la Matière Molle, 10 , rue Vauquelin, 75231, Paris Cedex 05, France.

${ }^{3}$ Global Station for Soft Matter, Global Institution for Collaborative Research and Education, Hokkaido University, Sapporo, Japon.

influencent les propriétés viscoélasJean-luc.desseyn@inserm.fr
Le mucus est un gel viscoélastique complexe qui recouvre les épithéliums sécrétoires qu'il protège des particules étrangères et des organismes pathogènes. La formation du gel, aussi appelée gélification, est largement attribuée aux mucines gélifiantes, des macromolécules sécrétées sous la forme de polymères fortement glycosylés qui captent et retiennent l'eau. Le mucus n'est pas un compartiment statique : il peut subir des modifications de ses propriétés qui dépendent de l'état physiologique de l'individu. On parlera alors de plasticité du mucus. L'interactome des mucines gélifiantes est un facteur clé des propriétés viscoélastiques du mucus [1]. De nombreux paramètres physico-chimiques modulent cette viscoélasticité du mucus en agissant sur la structure du réseau de mucines. Après avoir exposé la structure des mucines gélifiantes, nous détaillerons les mécanismes impliqués dans leur association entre elles, ou avec d'autres composants du mucus, pour former un gel viscoélastique dont nous décrirons les fonctions. Enfin, les différents paramètres physico-chimiques qui

Vignette (Photo @ Inserm - Jean-Marie Zahm). tiques des gels de mucus seront abordés afin d'illustrer leur dynamique.

\section{Structure des mucines gélifiantes}

Les mucines constituent une famille de 0 -glycoprotéines qui regroupe les mucines membranaires, qui participent à la structure du glycocalyx, et les mucines gélifiantes sécrétées par les cellules caliciformes, les glandes sous-muqueuses et les cellules séreuses [2]. Considérant la contribution mineure des mucines membranaires dans la viscoélasticité du mucus, cette revue se focalisera sur les mucines gélifiantes, que nous nommerons mucines dans la suite du texte. Chez l'homme, 5 mucines, appelées MUC2, MUC5AC, MUC5B, MUC6 et MUC19, ont été découvertes. Chacune possède un orthologue murin. Les gènes MUC2, MUC5AC, MUC5B et MUC6 sont organisés en cluster au sein du locus 11 pl5.5 tandis que MUC19 est localisé en 12q12. Ces gènes codent les squelettes peptidiques des mucines, encore appelés apomucines, dont la structure comporte 3 régions subdivisées en domaines (Figure 1).

\section{L'apomucine}

Les régions amino- et carboxy-terminales des mucines sont homologues au facteur von Willebrand ${ }^{1}$ avec lequel elles partagent un ancêtre com-

\footnotetext{
${ }^{1}$ Une glycoprotéine intervenant dans l'hémostase primaire.
} 


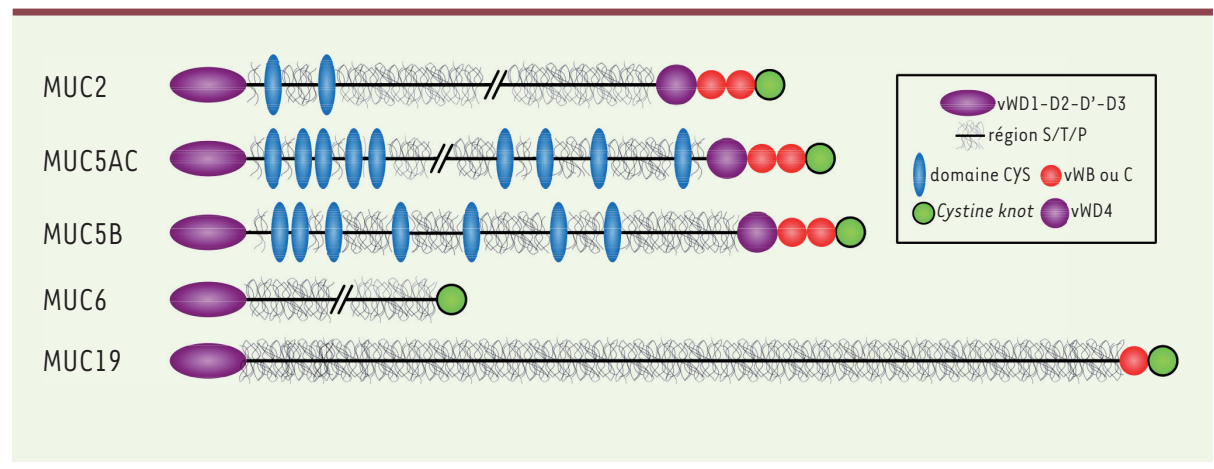

Figure 1. Structure des mucines gélifiantes. La région amino-terminale comprend les domaines vW (von Willebrand) D1, vWD2, vWD' et vWD3 (violet). La région centrale riche en chaînes 0 -glycosidiques (gris) est interrompue par les domaines hydrophobes Cys (bleu) pour les mucines MUC2, MUC5AC et MUC5B. L'extrémité carboxy-terminale comprend les domaines vWD4 (violet), vWB et vWC

(rouge) et CK (cystine knot, vert). MUC19 ne possède que vWC. MUC6 ne possède ni vWB, ni vWC. Les interruptions dans les apomucines représentent les polymorphismes de type VNTR (variable number of tandem repeats).

mun [3]. La région amino-terminale des mucines est en effet composée de 3 domaines $D$ du facteur von Willebrand (vWD1, vWD2 et vWD3) et d'un domaine $D$ tronqué (vWD'), entre vWD2 et vWD3. L'architecture de la partie carboxy-terminale est moins conservée entre les mucines. Elles possèdent toutes un domaine CK (cystine knot), constitué d'environ 80 acides aminés, de structure tridimensionnelle similaire à celle du TGF- $\beta$ (transforming growth factor $\beta$ ). En amont de ce domaine, un quatrième domaine D (vWD4) est retrouvé dans MUC2, MUC5AC et MUC5B. Il est suivi par un domaine $B(v W B)$ et un domaine $C(v W C)$ également présent dans le facteur von Willebrand. MUC19 ne possède pas de domaine $\mathrm{vWB}$ et MUC6 ne présente ni domaine vWB, ni domaine vWC, probablement à la suite d'une perte d'exon au cours de l'évolution (Figure 1).

La partie centrale des mucines est riche en résidus sérine, thréonine et proline, d'où son nom de région $S / T / P$. Ce sont les résidus sérine et thréonine qui portent les nombreuses chaînes 0 -glycosidiques des mucines. Chez l'homme, cette région est codée par des motifs répétés en tandem, organisés en un seul et unique grand exon de plusieurs kilobases. Ces motifs répétés, leurs tailles et leurs séquences, sont caractéristiques de chaque mucine. Ils ne sont pas toujours conservés entre espèces $[4,5]$. À l'exception de celle de MUC5B, cette région présente un polymorphisme de type VNTR (variable number of tandem repeats) intra- et inter-individuel [3].

MUC2, MUC5AC et MUC5B ont la caractéristique d'avoir des régions $\mathrm{S} / \mathrm{T} / \mathrm{P}$ interrompues par des domaines CYS. Le domaine CYS est constitué de 110 acides aminés dont 10 résidus cystéine qui sont conservés chez les vertébrés supérieurs. Ces cystéines seraient engagées dans cinq ponts disulfures intra-chaîne, qui confèrent à la protéine une structure secondaire globulaire [1]. Très conservé dans l'évolution, ce domaine est toujours présent en plusieurs copies dans les mucines: 2 dans MUC2, 9 dans MUC5AC et 7 dans MUC5B [6]. Sa séquence est majoritairement composée d'acides aminés hydrophobes. Ceci se traduit dans l'apomucine par une alternance entre les domaines cys hydrophobes et les régions S/T/P glycosylées qui sont hydrophiles. Des interactions hydrophobes réversibles entre mucines (mucine::mucine) dans l'environnement aqueux du mucus pourraient donc exister. Afin d'appréhender les fonctions du domaine CyS, une souris transgénique qui sécrète dans la lumière intestinale, avec MUC2, une protéine recombinante composée de 12 domaines CyS consécutifs a été produite. Chez ces souris, l'observation par immunohistochimie de coupes transversales du tissu colique a révélé un mucus mieux conservé en comparaison de celui de souris de type sauvage. Le mucus des souris transgéniques est également plus résistant à la pénétration, comme en témoigne sa moindre perméabilité, in vitro, à des billes carboxylées. In vivo, ces souris sont plus résistantes à une colite chimiquement induite et à des infections bactériennes expérimentales. On note également chez ces souris une plus grande expression de glycannes sialylés et sulfatés, respectivement dans le côlon et l'iléon, et, dans l'iléon, une augmentation de la flore lactobacillaire (une flore bénéfique dans le cas d'inflammation ou d'infection). Il semble donc que le domaine cys ait un rôle important dans les propriétés fonctionnelles du mucus, comme la protection de la muqueuse contre les particules étrangères et les pathogènes $[7,8](\rightarrow)$.

$(\rightarrow)$ Voir la Nouvelle de J.L. Desseyn et al., $\mathrm{m} / \mathrm{s} \mathrm{n}^{\circ} 12$, décembre 2015, page 1063

\section{La glycosylation des mucines gélifiantes}

La fraction glycannique constitue plus de $80 \%$ de la masse sèche des mucines. Ces sucres sont essentiellement des chaînes 0 -glycosylées, dont la taille varie entre 6 et 18 monosaccharides. Elles sont majoritairement situées dans les régions $\mathrm{S} / \mathrm{T} / \mathrm{P}$ de la glycoprotéine [9]. Cette 0 -glycosylation est en fait responsable de la structure en écouvillon qui est caractéristique des mucines (Figure 2). Elle est initiée dans l'appareil de Golgi par la liaison d'un résidu de $N$-acétylgalactosamine ( $\mathrm{GalNac}$ ) sur les groupes hydroxyles libres des résidus sérine et thréonine. D'autres monosaccharides, comme le galactose et la $\mathrm{N}$-acétylglucosamine, sont ensuite greffés à la chaîne afin de former le «noyau » des chaînes glycanniques. Des sucres sont ensuite ajou- 


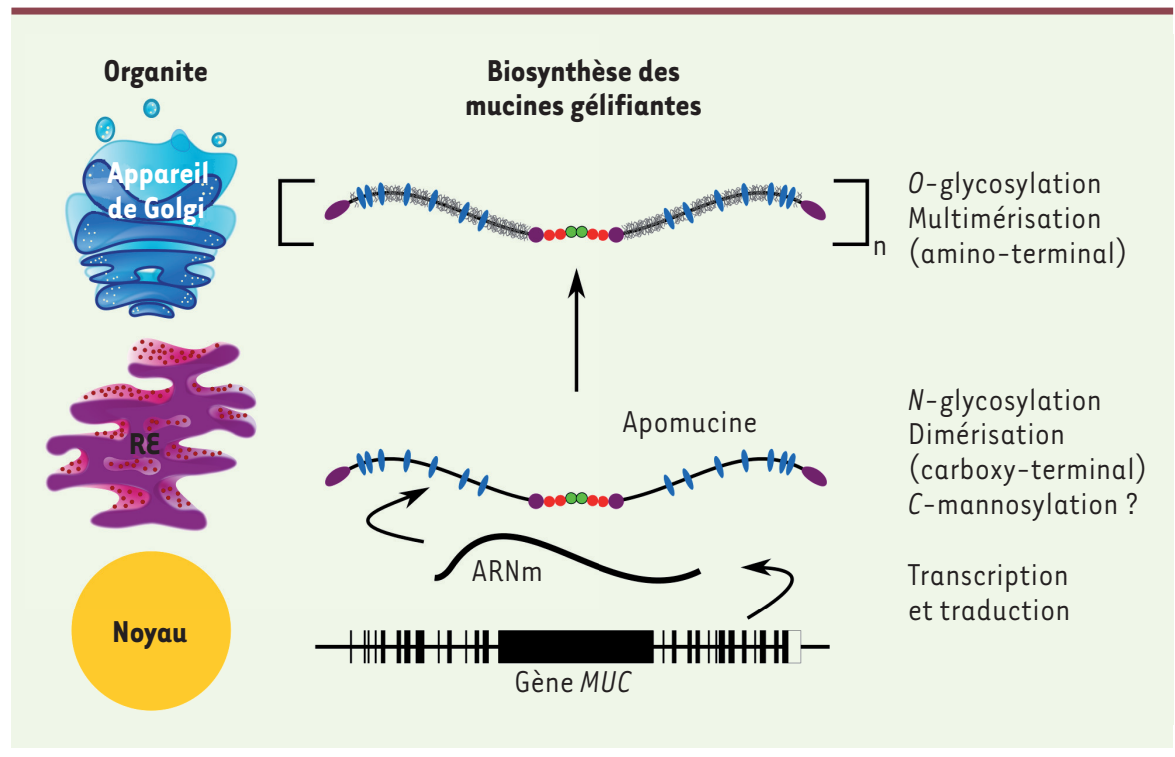

Figure 2. Biosynthèse des mucines gélifiantes. Les différentes étapes de la biosynthèse des mucines gélifiantes selon les compartiments cellulaires sont schématisées. RE : réticulum endoplasmique (reproduit et adapté avec la permission de [1]).

cellules muco-sécrétantes. Cette conformation condensée est rendue possible après électro-neutralisation des glycannes électronégatifs grâce à l'environnement physicochimique du granule, riche en calcium et au $\mathrm{pH}$ acide. La structure moléculaire des mucines peut être modifiée après leur sécrétion par tés, la chaîne se terminant alors par des déterminants sucrés variés, comme les antigènes de groupes sanguins [10]. L'ajout de fucose, d'acide sialique ou de groupements sulfate, participe aussi à la grande diversité intra- et inter-individuelle du profil de glycosylation des mucines.

Des sites de $\mathrm{N}$-glycosylation sont également présents dans les régions amino- et carboxy-terminales des mucines. La fonction de cette $N$-glycosylation est de faciliter, sans être indispensable, l'étape d'oligomérisation des mucines [11].

La $C$-mannosylation est une modification co-traductionnelle impliquant la liaison du carbone anomérique d'un $\alpha$-D-mannose au carbone C2 du cycle indole du premier tryptophane (Trp) de la séquence consensus Trp-Xaa-Xaa-Trp (où Xaa représente un acide aminé). Cette mannosylation particulière a été mise en évidence sur différentes molécules, toutes sécrétées. Elle reste toutefois controversée dans le cas des mucines. Elle pourrait jouer un rôle dans le trafic intracellulaire des mucines.

\section{Structure et fonctions du mucus}

\section{L'interactome des mucines}

\section{Les liaisons covalentes entre mucines}

La polymérisation des mucines est initiée dans le réticulum endoplasmique, où elles forment des dimères assemblés par des ponts disulfures entre leurs domaines carboxy-terminaux (CK) [12]. Ces dimères sont ensuite adressés au réseau trans-golgien où ils sont assemblés, via leur région amino-terminale, pour former de longs polymères linéaires (Figure 2). Le profil de multimérisation de cette région amino-terminale, déterminé in vitro à partir de cultures de cellules, pourrait être différent d'une mucine à l'autre. En effet, MUC5B et MUC5AC forment des dimères, alors que MUC2 des trimères [13-15]. Une fois polymérisées, les mucines sont condensées puis stockées dans des granules de sécrétion accumulés dans la région sous-apicale des

des protéases extracellulaires [16] ou des enzymes, comme la transglutaminase 2 [17].

\section{Les liaisons réversibles entre mucines}

Afin d'adapter les fonctions du mucus aux besoins physiologiques de l'individu, la structure du réseau de mucines peut être modifiée de manière réversible par des interactions de faible énergie. La nature de ces interactions entre mucines peut être déterminée par l'analyse de la réponse du mucus à une contrainte (un cisaillement ou une oscillation) de fréquence (entre généralement $10^{-2}$ et $10^{2}$ radians par seconde) ou d'intensité contrôlée. La contrainte exercée sur le fluide tend à désorganiser sa structure. Cependant, dans le cas de fluides viscoélastiques comme le mucus, sa composante élastique tend à ramener sa structure moléculaire à sa conformation initiale. À de basses fréquences ou intensités de contrainte, le mucus est dominé par des forces élastiques. Ainsi, après chaque déformation, la structure moléculaire du mucus retrouve sa conformation initiale. En augmentant la fréquence ou l'intensité de la contrainte, la structure du mucus se désorganise mais ne peut se reformer; la viscosité domine alors l'élasticité, et le gel s'écoule. Ce point de rupture a été évalué à 187 pascal ( $\mathrm{Pa}$ ) pour le mucus gastrique. En diminuant la fréquence ou l'intensité de la contrainte, le phénomène inverse se produit. Le mucus reforme alors un gel lorsque la force de cisaillement devient inférieure à $180 \mathrm{~Pa}$. Ce processus est appelé recouvrement. Le fait que la force exercée pour obtenir le point de rupture et le recouvrement soient quasi-identiques montre que le mucus est un gel physique qui est en partie maintenu par des interactions réversibles $[18,19]$. 
Des forces hydrophobes entre les régions amino-terminales de MUC5B, et des interactions avec les ions calcium ont récemment été mises en évidence. Ces interactions réversibles, car non covalentes, pourraient être impliquées dans le maintien de la structure compacte de la mucine au sein du granule de sécrétion [14, 20]. Le domaine CyS pourrait également participer à ces interactions réversibles entre mucines, en fonction par exemple de facteurs modulant leur structure comme la concentration calcique et le $\mathrm{pH}$ [21].

\section{Des composants «non-mucine » qui interagissent avec les mucines gélifiantes}

De nombreuses molécules «non-mucine » semblent intervenir dans la structure supramoléculaire des mucines, les principales pouvant être des peptides en trèfle (TFF), des lipides, le FcGBP (Fc fragment of Ig [immunoglobulin] G binding protein) et les transglutaminases.

Chez l'homme, les TFF forment une famille constituée de trois peptides (TFF1, TFF2 et TFF3), tous sécrétés par les cellules épithéliales. Leur nom vient de leur structure secondaire qui forme trois boucles maintenues par des ponts disulfures intra-chaîne. TFFl et TFF3 possèdent un seul domaine en trèfle, TFF2 deux. TFFl et TFF3 sont retrouvés sous la forme d'homodimères ou de complexes, avec des protéines qui ne sont pas encore identifiées. Les peptides en trèfle sont surtout exprimés dans le tractus digestif où ils s'associent avec les mucines par l'intermédiaire de différents types d'interactions (hydrophobe, ionique). Ils participent ainsi aux propriétés viscoélastiques du mucus [22]. Le rôle de ces peptides n'est pas encore totalement compris, mais des études suggèrent des fonctions de protection et de réparation de l'épithélium intestinal. La fraction lipidique du mucus est estimée à 1 à $2 \%$ de sa masse. Il s'agit surtout de lipides neutres et, dans une moindre mesure, de glycolipides et de phospholipides. Ces lipides créent une barrière hydrophobe protectrice à la surface du mucus. Ainsi, les lipides tensioactifs, comme la phosphatidylcholine, ont été associés avec une augmentation de la clairance mucociliaire dans l'arbre respiratoire. Ils fournissent également une meilleure protection contre une colite induite chimiquement [23]. À la surface de l'œil, les lipides forment une barrière protectrice en limitant l'évaporation du film lacrymal.

La FcGBP est exprimé dans l'intestin, le placenta et l'appareil reproducteur féminin. Elle est constituée d'une répétition en tandem de douze domaines homologues de type vWD. Elle se lie de manière covalente à MUC2 [24], mais I'hydrophobicité de FcGBP suggère également la possibilité de liaisons réversibles non covalentes avec les peptides hydrophobes des mucines. FcGBP pourrait favoriser la réparation du tissu intestinal ou capter les virions de VIH (virus de l'immunodéficience humaine) présents au sein des muqueuses. Par ses propriétés structurales et fonctionnelles, FcGBP est ainsi impliquée dans l'immunité des muqueuses, apportant un point d'ancrage aux IgG spécifiques de pathogènes.

Les transglutaminases constituent une famille d'enzymes ubiquitaires qui catalyse la formation de liaisons $\varepsilon(\gamma$-glutamyl)-lysine entre une lysine donneuse et une glutamine acceptrice. Ces enzymes permettent la formation de structures supramoléculaires en stabilisant les structures protéiques labiles. La transglutaminase 2 pourrait ainsi créer des liaisons isopeptidiques entre les domaines CyS de MUC2, au sein du granule de sécrétion. Cette enzyme apparaît donc importante pour la réticulation du gel, tout en permettant une meilleure protection des mucines contre les activités protéolytiques [17].

\section{Les fonctions du mucus}

Le mucus est très conservé chez les organismes métazoaires, suggérant des fonctions biologiques importantes mais qui dépendent néanmoins du mode de vie de l'organisme [1]. Chez l'homme, chaque tissu muco-sécrétant a un profil d'expression des mucines qui lui est propre (Tableau l). Les mucines ont donc probablement des fonctions différentes. Les fonctions, communes aux différents gels de mucus sont d'hydrater et de lubrifier les épithéliums qu'ils recouvrent [25], mais selon le tissu, des fonctions plus spécifiques leur sont attribuées.

Si le mucus oculaire piège avant tout les poussières et les potentiels pathogènes afin de les éliminer via le conduit naso-lacrymal, sa nature visqueuse permet également de dissiper l'énergie engagée lors du battement de paupières, prévenant ainsi les dommages de la cornée causés par des frictions répétées [26].

À la surface de la trachée, des bronches et de l'oreille moyenne, le battement de millions de cils évacue en permanence le mucus dans lequel les pathogènes sont piégés [1].

Dans la cavité utérine, le fœtus est protégé des infections vaginales par un bouchon de mucus qui se forme dans le canal endocervical. Des études récentes montrent que des défauts de ce bouchon sont associés à la survenue de naissances prématurées [27].

Le mucus recouvre également le tractus digestif, de la bouche au côlon. Dans la bouche, il permet la lubrification du bol alimentaire et facilite son transfert vers l'estomac. Dans l'estomac, il forme des couches distinctes, avec un gradient de $\mathrm{pH}$ qui protège l'épithélium d'une auto-digestion acide. Un gel plus fin couvre l'intestin grêle. II forme à ce niveau une barrière semi-perméable sélective, permettant le passage de nutriments de la lumière vers le tissu, mais qui est imperméable aux pathogènes. Dans le côlon, le mucus n'est pas un gel inerte homogène. II forme en effet deux gels distincts extrêmement organisés en de nombreuses sous-couches présentant des mucines différemment 0 -glycosylées [28]. Toujours dans le côlon, un gel adhérent, au contact du tissu, est surmonté d'une couche nonadhérente qui constitue, selon Johansson et al. [29], la niche écologique du microbiote. Dans le modèle de Kamphuis et al. [30], il serait au contact des fèces pour faciliter leur transit et contenir le microbiote. 


\begin{tabular}{|c|c|c|c|c|c|c|c|c|c|c|}
\hline & \multicolumn{6}{|c|}{ Mucines avec domaines CyS } & \multicolumn{4}{|c|}{ Mucines sans domaine Cys } \\
\hline & \multicolumn{2}{|c|}{ MUC2 } & \multicolumn{2}{|c|}{ MUC5AC } & \multicolumn{2}{|c|}{ MUC5B } & \multicolumn{2}{|c|}{ MUC6 } & \multicolumn{2}{|c|}{ MUC19 } \\
\hline & ARNm & Prot & ARNm & Prot & ARNm & Prot & ARNm & Prot & ARNm & Prot \\
\hline Glandes salivaires & & & + & + & & +++ & & & $?$ & $?$ \\
\hline Estomac & & + & +++ & +++ & & & +++ & +++ & & \\
\hline Duodénum/Iléon & +++ & +++ & & & & & +++ & & & \\
\hline Côlon & +++ & +++ & & & & & & & & \\
\hline Vésicule biliaire & & & & + & & +++ & & + & & \\
\hline Cavité nasale & & & + & & +++ & & + & & & \\
\hline Trachée & & & +++ & +++ & +++ & +++ & & & & \\
\hline Poumons & + & + & +++ & +++ & +++ & +++ & & & & \\
\hline Liquide séminal & & & & & & +++ & & +++ & & \\
\hline Prostate & & $?$ & & & & & & & & \\
\hline Vagin & & & & +++ & & & & & & \\
\hline Cervix & + & + & + & + & +++ & +++ & +++ & +++ & & \\
\hline Larynx/Pharynx & & $?$ & & & & & & & & \\
\hline Oreille moyenne & + & & +++ & +++ & +++ & +++ & & & + & + \\
\hline Conjonctive/Larmes & & + & & +++ & +++ & + & + & & +++ & +++ \\
\hline
\end{tabular}

Tableau I. Expression des mucines gélifiantes humaines chez l'individu sain. Pour chaque tissu muco-sécrétant, l'expression des mucines gélifiantes selon la littérature est indiquée au niveau ARN (transcription) ou protéique (Prot). Ce tableau met en évidence le fait que tous les tissus muco-sécrétants produisent au moins une mucine contenant des domaines CyS. +++ Mucine majeure ; + Mucine mineure ; ? Expression à confirmer

\section{Des paramètres qui influencent la viscoélasticité du mucus}

\section{L'environnement ionique}

Au sein du granule de sécrétion, la concentration importante d'ions calcium permet aux polymères linéaires de la mucine MUC5B d'adopter une conformation condensée par des interactions ioniques réversibles. Après leur sécrétion, les interactions ioniques des mucines avec les ions calcium sont remplacées par des interactions reposant sur des ions sodium. Ceci induit une hyper-osmolarité du mucus qui génère un mouvement d'eau vers le réseau de mucines avec une expansion rapide du réseau jusqu'à ce que l'équilibre de Donnan ${ }^{2}$ soit établi [31]. Les résidus sulfates et acides sialiques électronégatifs des mucines amplifient ce phénomène d'expansion par des répulsions électrostatiques. In fine, les mucines forment un réseau enchevêtré très hydraté à l'origine de la viscoélasticité du mucus [32] (Figure 3). In vitro, une augmentation de l'osmolarité du mucus par apport de chlorure de sodium $(\mathrm{NaCl})$ provoque une augmentation de sa viscoélasticité, en réduisant les répulsions électrostatiques et en stabili-

\footnotetext{
${ }^{2}$ L'équilibre de Donnan représente à la fois des mouvements d'eau (osmose) et d'ions (transports actifs et passifs) afin d'équilibrer les concentrations et les charges entre deux compartiments, ici les compartiments aqueux et le gel de mucus.
}

sant les interactions hydrophobes entre mucines [33]. Les ions carbonates $\left(\mathrm{HCO}_{3}^{-}\right)$extracellulaires captent les ions calcium du granule de sécrétion, ce qui facilite le déploiement du réseau de mucines à la surface des épithéliums et donc la formation d'un gel viscoélastique [34].

\section{L'influence du pH}

II existe une forte corrélation entre les propriétés viscoélastiques du mucus et son pH. Cela a été particulièrement étudié dans le mucus gastrique où le changement drastique de $\mathrm{pH}$ durant la digestion induit, à $\mathrm{pH} 4$, une transition de viscoélasticité du mucus depuis l'état solution (viscosité > élasticité) vers l'état gel (élasticité > viscosité). Les mécanismes moléculaires impliquent une rupture des ponts salins ainsi qu'un changement conformationnel des mucines à ce $\mathrm{pH}$ acide. Cela favorise les interactions entre les parties hydrophobes des mucines et augmente la réticulation du gel [35]. La structure à l'origine de ce phénomène pourrait être le domaine Cys. Ceci est en effet suggéré par l'étude des propriétés du mucus cervico-vaginal, 


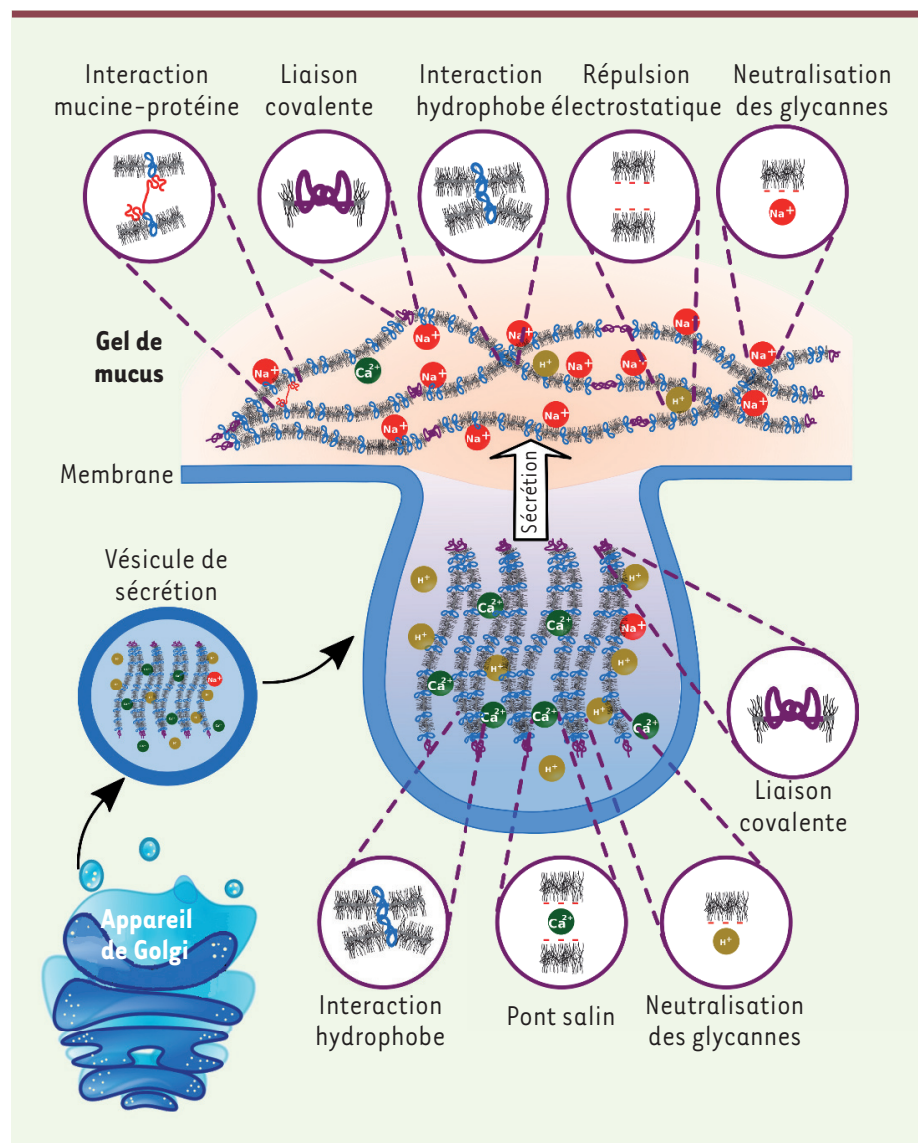

Figure 3. Représentation schématique des interactions entre mucines gélifiantes dans le granule de sécrétion et dans le mucus. Les mucines gélifiantes sont compactées dans les granules par neutralisation des déterminants négatifs portés par les glycannes. Le calcium permet la formation de ponts salins. Le $\mathrm{pH}$ acide provoque un changement conformationnel des mucines, qui expose leurs parties hydrophobes et amplifie le phénomène de compaction. Dans le compartiment extracellulaire, le calcium est remplacé par du sodium, à l'origine de l'expansion rapide du réseau de mucines par équilibre de Donnan. Le réseau adopte une conformation enchevêtrée stabilisée par des interactions covalentes et réversibles (reproduit avec la permission de [1]).

qui varient selon le cycle ovulatoire et dont la structure semble indépendante du $\mathrm{pH}$ en phase péri-ovulatoire [36, 37].

\section{Concentration des mucines gélifiantes}

L'équation de Fox-Flory ${ }^{3}$ décrit le passage d'un fluide à l'état solide vitreux lors d'une diminution rapide de sa température : plus les polymères qui composent ce fluide auront une masse moléculaire importante et/ou seront réticulés, plus cette transition sera précoce. La structure et la composition des gels de mucus sont donc des facteurs clés pour l'acquisition de ses propriétés viscoélastiques. II a été montré, par analyse de la diffusion de particules dans le gel, que les mucines forment un gel à partir

${ }^{3}$ Formule empirique simple reliant la masse molaire moyenne d'un polymère à sa température de transition vitreuse. d'une concentration protéique de $10 \mathrm{mg} / \mathrm{ml}$. La transition vers l'état visqueux, définie comme la concentration à partir de laquelle les mucines s'associent en un réseau protéique enchevêtré, ne s'opère qu'autour de 20 à $30 \mathrm{mg} /$ $\mathrm{ml}$ [38]. Pour des concentrations de mucines élevées, un gel se forme, malgré la présence d'agents chaotropiques. À ces concentrations, les chaînes glycanniques des mucines forment des inter-digitations qui augmentent la viscosité du gel. Ce lien entre réticulation et viscoélasticité du mucus est exploité afin de concevoir des agents mucolytiques capables de dépolymériser les mucines et diminuer la viscosité trop élevée des gels de mucus dans certaines pathologies comme la mucoviscidose.

\section{Les autres facteurs}

Des partenaires protéiques, tels les TFF, stabilisent les liaisons inter-mucines et contribuent à la viscoélasticité du mucus. Chez la femme, la concentration de TFF3 dans le bouchon de mucus cervical, spontanément éjecté au moment de l'accouchement, est corrélée à la viscosité et l'élasticité du gel [24].

\section{Conclusion}

La production des différents domaines de mucines par la technique de l'ADN recombinant permet de définir le rôle de chacun d'entre eux dans la structure et les fonctions du mucus. La polymérisation des mucines dues aux liaisons covalentes qu'elles forment est maintenant bien caractérisée. II apparaît cependant désormais que des forces de faible énergie participent également à la viscoélasticité et à la plasticité des gels de mucus [21]. La nature labile de ces interactions, ainsi que leur dépendance à l'environnement physico-chimique du mucus, rendent leur étude complexe [37]. L'identification des domaines protéiques et des paramètres physico-chimiques impliqués dans ces interactions réversibles permettrait, à terme, de pouvoir moduler les propriétés de ces gels en modifiant le réseau de mucines [7]. Ceci ouvrirait de nouvelles pistes thérapeutiques afin de pouvoir renforcer le mucus intestinal pour, par exemple, les maladies inflammatoires chroniques de l'intestin, ou, à l'inverse, pour en diminuer la réticulation, dans le poumon des individus atteints de mucoviscidose. $\diamond$

\section{SUMMARY}

Gel-forming mucins structure governs mucus gels viscoelasticity

Mucus is the first line of innate mucosal defense in all mammals. Gel-forming mucins control the rheological properties of mucus hydrogels by forming a network in which hydrophilic and hydrophobic regions coexist, and it has been revealed that the network is formed 
through both covalent links and reversible links such as hydrophobic interactions in order to modulate the structure as a function of the physiological necessities. Here, we review the structure and functions of the mucus in terms of the gel-forming mucins protein-protein interactions, also called interactome. Since it is difficult to characterize the low energy reversible interactions due to their dependence on physico-chemical environment, their role is not well understood. Still, they constitute a promising target to counteract mucus abnormalities observed in mucus-associated diseases. $\diamond$

\section{REMERCIEMENTS}

B.D. est soutenu par une allocation de doctorat de la région Hauts-de-France et du Centre Hospitalo-Universitaire de Lille. Ce travail a reçu le soutien de la région Hauts-de-FranceContrat : ARCIr Volet Dynamique 1400324.

\section{LIENS D'INTÉR̂ิT}

Les auteurs déclarent n'avoir aucun lien d'intérêt concernant les données publiées dans cet article.

\section{RÉFÉRENCES}

1. Demouveaux B, Gouyer V, Gottrand F, et al. Gel-forming mucin interactome drives mucus viscoelasticity. Adv Colloid Interface Sci $2018 ; 252: 69-82$.

2. Ma J, Rubin BK, Voynow JA. Mucins, mucus, and goblet cells. Chest 2018 ; 154 : 169-76.

3. Desseyn JL, Aubert JP, Porchet N, et al. Evolution of the large secreted gel-forming mucins. Mol Biol Evol $2000 ; 17: 1175-84$.

4. Desseyn JL, Clavereau I, Laine A. Cloning, chromosomal localization and characterization of the murine mucin gene orthologous to human MUC4. EurJ Biochem $2002 ; 269: 3150-9$.

5. Desseyn JL, Laine A. Characterization of mouse Muc6 and evidence of conservation of the gelforming mucin gene cluster between human and mouse. Genomics 2003 ; $81: 433-6$.

6. Desseyn JL. Mucin CyS domains are ancient and highly conserved modules that evolved in concert. Mol Phylogenet Evol $2009 ; 52: 284-92$.

7. Gouyer V, Dubuquoy L, Robbe-Masselot C, et al. Delivery of a mucin domain enriched in cysteine residues strengthens the intestinal mucous barrier. Sci Rep $2015 ; 5: 9577$.

8. Desseyn JL, Gouyer V, Gottrand F. Modification à façon des propriétés physiques du mucus : preuve de concept et applications potentielles. Med Sci (Paris) 2015 ; 31 : 1063-6.

9. Robbe-Masselot C, Capon C, Maes $\varepsilon$, et al. Evidence of regio-specific glycosylation in human intestinal mucins: presence of an acidic gradient along the intestinal tract. J Biol Chem 2003; $278: 46337-48$.

10. Corfield AP. Mucins: a biologically relevant glycan barrier in mucosal protection. Biochim Biophys Acta $2015 ; 1850: 236-52$

11. Asker N, Axelsson MAB, Olofsson S0, et al. Dimerization of the human MUC2 mucin in the endoplasmic reticulum is followed by a $\mathrm{N}$-glycosylation-dependent transfer of the mono- and dimers to the Golgi apparatus. J Biol Chem 1998 ; 273 : 18857-63.

12. Bell SL, Xu G, Forstner JF. Role of the cystine-knot motif at the $\mathrm{C}$-terminus of rat mucin protein Muc2 in dimer formation and secretion. Biochem J 2001 ; $357: 203-9$.

13. Sheehan JK, Kirkham S, Howard M, et al. Identification of molecular intermediates in the assembly pathway of the MUC5AC mucin. J Biol Chem 2004 ; 279 : 15698-705.

14. Ridley CE, Kouvatsos N, Raynal B, et al. Assembly of the respiratory mucin MUC5B: a new model for a gel-forming mucin. J Biol Chem $2014 ; 289$ : 16409-20.

15. Godl K, Johansson ME V, Lidell ME, et al. The $N$ terminus of the MUC2 mucin forms trimers that are held together within a trypsin-resistant core fragment. J Biol Chem $2002 ; 277: 47248-56$.

16. Wickström C, Carlstedt I. N-terminal cleavage of the salivary MUC5B mucin: analogy with the von Willebrand propolypeptide? J Biol Chem. 2001 ; 276 : 47116-21.
17. Recktenwald C V, Hansson GC. The reduction-insensitive bonds of the MUC2 mucin are isopeptide bonds. J Biol Chem 2016;291 : 13580-90.

18. McCullagh CM, Jamieson AM, Blackwell J, et al. Viscoelastic properties of human tracheobronchial mucin in aqueous solution. Biopolymers $1995 ; 35$ : 149-59.

19. Taylor C, Allen A, Dettmar PW, et al. The gel matrix of gastric mucus is maintained by a complex interplay of transient and nontransient associations. Biomacromolecules $2003 ; 4$ : 922-7.

20. Trillo-Muyo S, Nilsson HE, Recktenwald CV, et al. Granule-stored MUC5B mucins are packed by the non-covalent formation of $\mathrm{N}$-terminal head-tohead tetramers. J Biol Chem 2018 ; 293 : 5746-54.

21. Ridley CE, Kirkham S, Williamson SJ, et al. Biosynthesis of the polymeric gelforming mucin MUC5B. Am J Phys Lung Cell Mol Phys 2016; 310 : L993-1002.

22. Bastholm SK, Samson MH, Becher N, et al. Trefoil factor peptide 3 is positively correlated with the viscoelastic properties of the cervical mucus plug. Acta Obs Gynecol Scand $2017 ; 96: 47-52$.

23. Kovács $T$, Varga G, Erces D, et al. Dietary phosphatidylcholine supplementation attenuates inflammatory mucosal damage in a rat model of experimental colitis. Shock $2012 ; 38: 177-85$.

24. Harada N, lijima S, Kobayashi K, et al. Human IgGFc binding protein (FcgammaBP) in colonic epithelial cells exhibits mucin-like structure. J Biol Chem 1997 ; 272 : 15232-41.

25. Wang X, Du M, Han H, et al. Boundary lubrication by associative mucin. Langmuir $2015 ; 31: 4733-40$.

26. Hodges RR, Dartt DA. Tear film mucins: front line defenders of the ocular surface; comparison with airway and gastrointestinal tract mucins. Exp Eye Res $2013 ; 117: 62-78$.

27. Critchfield AS, Yao G, Jaishankar A, et al. Cervical mucus properties stratify risk for preterm birth. PLoS One 2013 ; 8 : e69528.

28. Gouyer V, Gottrand F, Desseyn JL. The extraordinarily complex but highly structured organization of intestinal mucus-gel unveiled in multicolor images. PLoS One 2011; 6 : el8761.

29. Johansson ME V, Larsson JMH, Hansson GC. The two mucus layers of colon are organized by the MUC2 mucin, whereas the outer layer is a legislator of host-microbial interactions. Proc Natl Acad Sci USA 2011 ; 108 : 4659-65.

30. Kamphuis JBJ, Mercier-Bonin $M$, Eutamène $H$, et al. Mucus organisation is shaped by colonic content; a new view. Sci Rep 2017 ; 7 : 8527.

31. Verdugo P. Supramolecular dynamics of mucus. Cold Spring Harb Perspect Med $2012 ; 2$ : a009597.

32. Davies HS, Singh P, Deckert-Gaudig T, et al. Secondary Structure and Glycosylation of Mucus Glycoproteins by Raman Spectroscopies. Anal Chem $2016 ; 88: 11609-15$.

33. Wagner C $\varepsilon$, Turner BS, Rubinstein M, et al. A rheological study of the association and dynamics of MUC5AC gels. Biomacromolecules 2017 ; 18 : 3654-64.

34. Muchekehu RW, Quinton PM. A new role for bicarbonate secretion in cervicouterine mucus release.J Physiol $2010 ; 588: 2329-42$.

35. Celli JP, Turner BS, Afdhal NH, et al. Rheology of gastric mucin exhibits a $\mathrm{pH}$-dependent sol-gel transition. Biomacromolecules $2007 ; 8$ : 1580-6.

36. Brunelli R, Papi M, Arcovito G, et al. Globular structure of human ovulatory cervical mucus. FASEB J. $2007 ; 21: 3872-6$.

37. Wang $Y-Y$, Lai SK, Ensign LM, et al. The microstructure and bulk rheology of human cervicovaginal mucus are remarkably resistant to changes in $\mathrm{pH}$. Biomacromolecules 2013; $14: 4429-35$.

38. Georgiades P, Pudney PDA, Thornton DJ, et al. Particle tracking microrheology of purified gastrointestinal mucins. Biopolymers $2014 ; 101$ : 366-77.

\section{TIRÉS À PART}

J.L. Desseyn

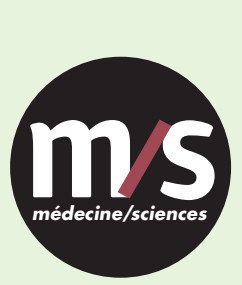

Tarifs d'abonnement $m / s-2018$

> Grâce à $\mathrm{m} / \mathrm{s}$, vivez en direct les progrès

des sciences biologiques et médicales

Abonnez-vous

à médecine/sciences

Bulletin d'abonnement page 890 dans ce numéro de $\mathrm{m} / \mathrm{s}$

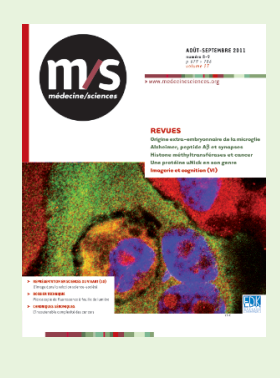

